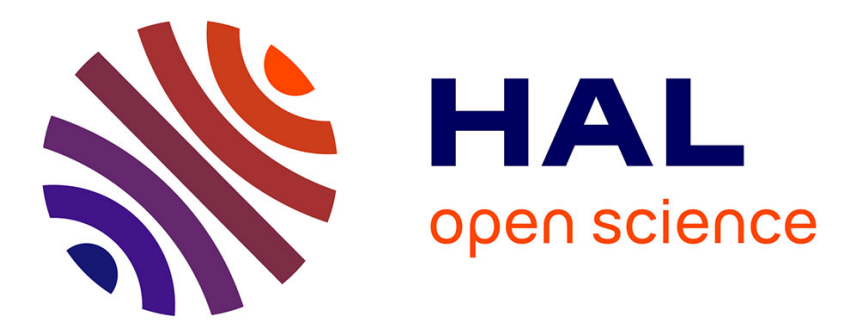

\title{
Optimal Consensus set for nD Fixed Width Annulus Fitting
}

Rita Zrour, Gaëlle Largeteau-Skapin, Eric Andres

\section{To cite this version:}

Rita Zrour, Gaëlle Largeteau-Skapin, Eric Andres. Optimal Consensus set for nD Fixed Width Annulus Fitting. Seventeenth International Workshop on Combinatorial Image Analysis, Nov 2015, Calcutta, India. pp.101-114, 10.1007/978-3-319-26145-4_8 . hal-01211471

\section{HAL Id: hal-01211471 \\ https://hal.science/hal-01211471}

Submitted on 5 Oct 2015

HAL is a multi-disciplinary open access archive for the deposit and dissemination of scientific research documents, whether they are published or not. The documents may come from teaching and research institutions in France or abroad, or from public or private research centers.
L'archive ouverte pluridisciplinaire HAL, est destinée au dépôt et à la diffusion de documents scientifiques de niveau recherche, publiés ou non, émanant des établissements d'enseignement et de recherche français ou étrangers, des laboratoires publics ou privés. 


\title{
DRAFT : Optimal Consensus set for $n \mathrm{D}$ Fixed Width Annulus Fitting
}

\author{
Rita Zrour, Gaelle Largeteau-Skapin, and Eric Andres \\ Université de Poitiers, Laboratoire XLIM, SIC, UMR CNRS 7252, BP 30179, \\ F-86962 Futuroscope Chasseneuil, France \\ \{rita.zrour, gaelle.largeteau.skapin,eric.andres\}@univ-poitiers.fr
}

\begin{abstract}
This paper presents a method for fitting a $n \mathrm{D}$ fixed width spherical shell to a given set of $n \mathrm{D}$ points in an image in the presence of noise by maximizing the number of inliers, namely the consensus set. We present an algorithm, that provides the optimal solution(s) within a time complexity $O\left(N^{n+1} \log N\right)$ for dimension $n, N$ being the number of points. Our algorithm guarantees optimal solution(s) and has lower complexity than previous known methods.
\end{abstract}

\section{Introduction}

Shape fitting is a general problem that is of very practical nature, namely extracting features from data with a high level of noise. This problem has been widely addressed in very different fields spanning from computer graphics and image processing to data mining in large dimensional databases [2]. In this paper we are looking into the problem of optimal fitting an $n \mathrm{D}$ annulus of fixed width in an image in the presence of outliers. The set of points which fits a model is called a consensus set. Note that an annulus in higher dimensions is sometimes referred to as $n$-sphere shell. We preferred to use the common denomination annulus for all dimensions.

This paper aims at finding the optimal consensus set (maximal number of inliers) inside a fixed width $n \mathrm{D}$ annulus, where the center and the radius are unknowns. Most annuli fitting methods try to minimize the thickness of the fitted annuli $[1$, $5,17]$. In our case, we are interested in digital circles and spheres and more specifically Andres digital circles and hyperspheres [3] or $k$-Flake digital circles-spheres [18]. In those cases, the thickness is directly linked with topological properties. The most common fitting methods are based on variants of the RANdom Sample Consensus (RANSAC) algorithm [8], which is a robust parameter estimation algorithm widely used in the field of computer vision. However RANSAC is inherently probabilistic in its approach and does not guarantee any optimality. In this paper we are looking for (all the) optimal solutions in order to generate base solutions to which we can compare and validate other, non exact, methods. It is also a problem when looking for multiple fittings in the same image. 
In [20] and [4], brute force algorithms were proposed to compute the optimal consensus set respectively for Andres digital circles [3] (defined as digital points inside a classical annulus of fixed width) and 0-Flake digital circles [18] (8connected circles) with a time complexity of $O\left(N^{4}\right)$ where $N$ is the number of points. A new method was proposed in [11] for fitting 0-Flake digital circles that has a complexity $O\left(N^{3} \log N\right)$. Our main contribution in this article is the extension of the fitting problem proposed in [20] to $n \mathrm{D}$ and reformulating it using a space transformation similar to the one proposed by [6] which was used in [11]. However the major difference with [6] is that the width is fixed and the parameter we want to maximize is the number of inliers.

The simpler problem of fitting circles and spheres is a largely studied problem. Most common approaches are based on least squares [15] or adapted Hough Transforms [10]. Those are however not adapted for annulus fitting. The circle fitting method proposed by O'Rourke et al. $[13,16]$ that transforms a circle separation problem into a plane separability problem, is also not well suited because the fixed width of the digital circles translates into non fixed vertical widths for the planes. In this case, the problem is complicated (See [14] for some ideas on how to handle this difficulty). For annuli detection, various approaches have been proposed. Most of these methods are probabilistic approaches that minimize the width of the annuli. Among these algorithms, some consider that no noise is present in the image, and concentrate only on the problem of recognition instead of the fitting problem $[1,5,17]$. However noise in real world is omnipresent in the input and so many algorithms dealing with outliers have been proposed $[7,9,12]$; in these algorithms, the number of outliers is usually predefined $[9,12]$ which is not always realistic.

The idea of the 2D algorithm is the following: given a set $S$, we consider all the annuli that have two specific points of $S$ on the border of the annulus. All the annuli centers with those two points on the border are then located on a straight line. This straight line is taken as a parametric axis. We then determine when a point of $S$ enters and leaves the annulus while the center moves along the axis. This allows us to compute the intervals where the number of inliers is maximized. By considering all the combinations of points, we are able to compute the exhaustive set of all optimal consensus sets in $O\left(N^{3} \log N\right)$. See [19] for a similar approach for line and plane fitting. The $n \mathrm{D}$ algorithm works in a similar way than the $2 \mathrm{D}$ algorithm [20]. However, we show that the annulus can de defined by $n$ specific points that are all on the external border. This characterization allows to greatly simplify both the proof of the characterization of annuli and the computation of the parametric axis defined by annuli centers. The final algorithm has an $O\left(N^{n+1} \log N\right)$ time complexity and leads to the exact optimal solutions for the problem of fixed width annulus fitting.

The paper is organized as follows: in Section 2 we expose the problem of annulus fitting and present some properties of the annuli with fixed width. Section 3 presents the dual space we use and provide the algorithm for finding the optimal $n \mathrm{D}$ annuli. Section 4 presents some 2D and 3D experiments. Finally Section 5 states some conclusion and perspectives. 


\section{Annulus fitting}

A $n \mathrm{D}$ annulus $A(C, R, \omega)$ of width $\omega$ and radius $R$ centered at $C\left(c_{1}, c_{2}, \ldots, c_{n}\right)$, is defined by the set of points in $\mathbb{R}^{n}$ satisfying two inequalities:

$$
A(C, R, \omega)=\left\{\left(p_{1}, p_{2}, \ldots, p_{n}\right) \in \mathbb{R}^{n}: R^{2} \leq \sum_{i=1}^{n}\left(p_{i}-c_{i}\right)^{2} \leq(R+\omega)^{2}\right\}
$$

where $C\left(c_{1}, c_{2}, \ldots, c_{n}\right) \in \mathbb{R}^{n}$ and $R, \omega \in \mathbb{R}^{+}$.

Using the above annulus model, our fitting problem is then described as follows: given a finite set $S=\left\{P_{i}, i \in[1, N], \in \mathbb{R}^{n}\right\}$ of $N n$ D points we would like to find the parameters (center and radius) of an annulus $A$ of given width $\omega$ that contains the maximum number of points in $S$. Points $P_{i} \in S \cap A$ in $n \mathrm{D}$ are called inliers; otherwise they are called outliers. We also say that the annulus $A$ covers the set $S \cap A$. We denote $B_{i}(C, R)$ (respectively $B_{e}(C, R+\omega)$ ) the internal (resp. external) border of the annulus $A(C, R, \omega)$, i.e. the set of points located at distance $R$ (resp. $R+\omega$ ) from $C$.

\subsection{Annulus Characterization}

Our approach is focused on inlier sets, also called consensus sets. Since $S$ is finite, the number of different consensus sets for the annulus is finite as well although too big to consider them all. However, as we are looking for the biggest consensus set(s), only the annuli that contain a minimum number of $n+1$ points, uniquely defining an annuli, are considered. For all the different consensus sets $\mathcal{C}$, with at least $n+1$ points from a given set $S$, we are going compute the size of each one. The center and radius are actually a side result of this search. We present in this section some characterization of $n \mathrm{D}$ annuli that will allow us to explore all those concensus sets and further on to build an optimal fitting algorithm for annuli.

\section{$2.2 n \mathrm{D}$ Annular characterizations}

In [20], we proposed a brute force algorithm for fitting fixed width annuli in 2D. We showed that if an optimal solution exists then there exists an equivalent optimal solution (with the same set of inliers) having three points on the border (internal and/or external). Testing all the configurations of three points and counting the inliers leads therefore to all the possible optimal solutions. This brute force method leads however to an $O\left(N^{4}\right)$ complexity for $N$ the number of points to fit. Its extension to $n \mathrm{D}$ is straight forward and needs $n+1$ points to be on the border (either internal or external), but it also leads to an $O\left(N^{n+2}\right)$ complexity.

In this article we need only $n$ points instead of $n+1$ points in $n \mathrm{D}$; however the restriction is that the $n$ points must be on the external border of the annulus. The 
last point is found in $O(N \log N)$ using a dual space, which will be presented in section 3.1. Using such a dual space, the algorithm has an overall time complexity of $O\left(N^{n+1} \log N\right)$. Note that we are looking for the exact optimal solutions, this complexity is, so far, the best to our knowledge to solve this problem.

The following theorem states that given an annulus $A$ of width $\omega$ covering a set of points $S$, with $|S| \geq n$, there exists at least one other annulus $A^{\prime}$ of same width, that also covers $S$ with at least $n$ points of $S$ on its external border $B_{e}$.

Theorem 1. Let $S$ be a finite set of $N(N \geq n)$ points in $\mathbb{R}^{n}$. Let $A=(C, R, \omega)$ be an annulus covering $S$. Then it exists $A^{\prime}=\left(C^{\prime}, R^{\prime}, \omega\right)$ covering $S$ such that:

$$
\exists ! Q_{1}, Q_{2}, \ldots, Q_{n} \in S \cap B_{e}\left(C^{\prime}, R^{\prime}+\omega\right) .
$$

a)

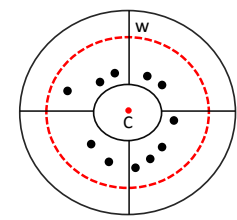

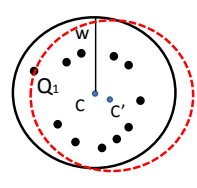

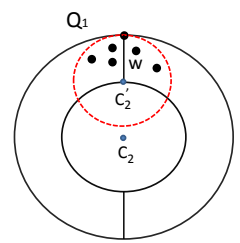

d)

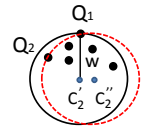

Fig. 1. Case A of the proof in 2D : a) While decreasing the radius of the annulus (colored black) to reach a first point $Q_{1}$ we may arrive to an internal radius of 0 (annulus in red) without any point on $B_{e}$, in this case a translation is needed as shown in b) to reach a point $Q_{1}$ on $B_{e}$. c) While Decreasing the radius of the annulus (colored black) by moving the center along axis $C_{2} Q_{1}$ to reach a second point $Q_{2}$, we may arrive to an internal radius of 0 and no point $Q_{2}$ on $B_{e}$ (annulus in red), in this case a rotation is done as shown in d) until reaching a second point $Q_{2}$ on $B_{e}$.

a)

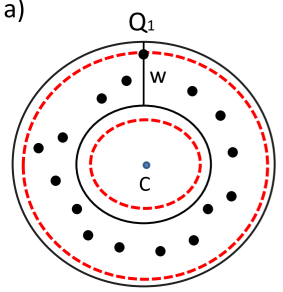

b)

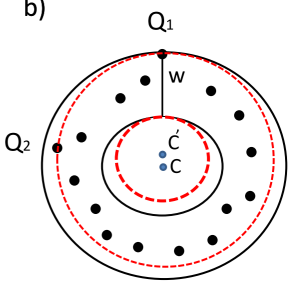

Fig. 2. Case B of the proof in $2 D$ : a) Decreasing the radius until reaching a point $Q_{1}$ on $B_{e}$. b) Decreasing the radius by moving the center along axis $C Q_{1}$ until reaching a second point $Q_{2}$. 
a)

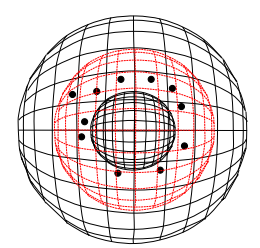

b)

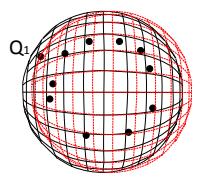

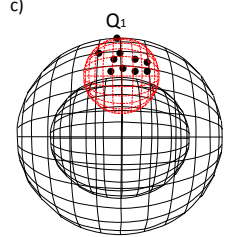

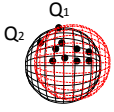

Fig. 3. Case A of the proof in $3 \mathrm{D}:$ a) While decreasing the radius it may lead to an internal radius of 0 without any point on $B_{e}$; in this case a translation is needed as shown in b) to reach a point $Q_{1}$ on $B_{e}$. c) Decreasing the radius while maintaining $Q_{1}$ on the border until reaching an internal radius of 0 ; in this case a rotation is needed as shown in d) in order to reach a point $Q_{2}$ on $B_{e}$.

a)

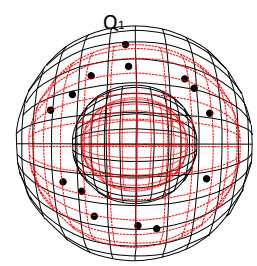

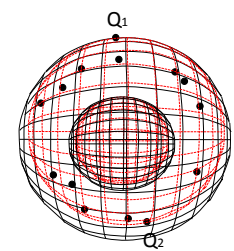

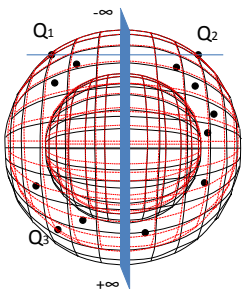

Fig. 4. Case B of the proof in $3 \mathrm{D}:$ a) Decreasing the radius until reaching a first point $Q_{1}$ on $B_{e}$. b) Decreasing the radius while maintaining $Q_{1}$ on the border until reaching a second point $Q_{2}$. c) Case B of the proof in 3D: moving the center along the bisector of $Q_{1} Q_{2}$ until reaching a third point $Q_{3}$.

Proof. Let $S$ be a finite set of $N(N \geq n)$ points in $\mathbb{R}^{n}$. Let $A=(C, R, \omega)$ be an annulus that covers $S$. The theorem proof is given as follows:

A. If the internal radius of the annulus is 0 , the problem is reduced to the problem of a hyper-sphere of fixed radius. The hypersphere can either be translated towards the closest point of $S$ if there are no point on the external border already (Figure 1a,b and Figure 3a,b) either it can be rotated around the axis formed by the already known points until reaching another point (Figure 1c,d and Figure 3c,d). This last step is done until $n$ points lie on $B_{e}$.

B. If the internal radius is greater than 0 , the building process consists in decreasing the radius while keeping the width fixed until reaching the points on the external border $B_{e}$ :

- If there is no point on the border, the radius is decreased while the center is fixed until reaching a point on $B_{e}$ (Figure 2a and Figure 4a).

- If there is one point $Q_{1}$ on the border, the radius is decreased while the center moves along the straight line $C Q_{1}$ towards $Q_{1}$ (which keep $Q_{1}$ on $B_{e}$ ) until reaching a second point on $B_{e}$ (Figure $2 \mathrm{~b}$, Figure $4 \mathrm{~b}$ ).

- If there are already $k>1$ points on $B_{e}$ then, we consider the barycenter $B$ of those $k$ points. We can now consider the straight line $\Delta$ passing through 
$B$ and $C$. By moving the center of the annulus along $\Delta$ towards $B$ we can reduce the radius of $B_{e}$ while keeping these $k$ points on $B_{e}$ (Figure $4 \mathrm{c}$ ). It should be noted that these steps are repeated until reaching $n$ points on $B_{e}$ or having an annulus with width 0 which leads to case A.

\section{Fitting algorithm}

Let us define an equivalence class of all the annuli that cover the same consensus set. We suppose that there are more than $n$ points in the image, otherwise all the points can be covered and the problem is somewhat trivial. The annulus characterization in Theorem 1 ensures that the optimal consensus set has always at least $n$ points. It also shows that we can always find a representative of the equivalent class with $n$ points of $S$ on the outer border. Let us find now, among the representatives of the equivalent classes of consensus sets with at least $n$ points, the annulus that cover(s) the maximum number of points of $S$.

The idea behind our fitting method is inspired by [6] where the authors maximize the width of an empty annulus in $2 D$. In [6], the authors look for the biggest empty annulus in a dual space based on the distance to the center. For each couple of points $\left(Q_{1}, Q_{2}\right)$, the possible positions for the center of a $2 \mathrm{D}$ annulus passing through $Q_{1}$ and $Q_{2}$ is the bisector of both points which then forms the abscissa axis of the dual space. Each other point is associated to a curve that represents its distance to the possible centers. Using this specific space, they find when each point enters or leaves the annulus. A sorting of these intervals leads to the maximal empty annulus passing through $Q_{1}$ and $Q_{2}$. A comparison among all the possible couples of points leads to the general result. In [6], the authors do not represent an annulus in their parameter space but only circles and the width $\omega$ of the annulus is maximized.

Our purpose in this work is different since, in our problem, we tried to maximize the number of inliers inside an annulus with fixed width, so we have two concentric circles with a fixed distance $\omega$ between them. Moreover, we have adapted this method to $n \mathrm{D}$. As we will see, their idea of taking the axes where the possible centers of the annuli are located can be adapted to our case. We first describe the dual space (Section 3.1) and then explain how we obtain the optimal consensus set(s) (Section 3.2).

\subsection{Dual space and annulus fitting in $n \mathrm{D}$}

According to Theorem 1 , in $n \mathrm{D}$, an annulus has at least $n$ unique critical points $Q_{1}, . ., Q_{n}$ located on its external border. Such an annulus has necessarily its center on a straight line, denoted $\Delta$, that passes through the barycenter $B$ of the points $Q_{1}, \ldots, Q_{n}$ and that is orthogonal to the hyperplane $H$ (of dimension $n-1$ ) induced by those same points (see Figure 5 a for an example in 2D). Let us define a $2 \mathrm{D}$ dual space as follows:

- The origin $O_{d u a l}(0,0)$ corresponds to the barycenter $B$ of the points $Q_{1}, . ., Q_{n}$. 
- The abscissa $X$ axis represents the possible locations of the center (it is a representative of $\Delta$ ): a center $C$ is associated to $C_{d u a l}(\operatorname{dist}(C, B), 0)$ (Figure $5 \mathrm{~b})$.

- The ordinate $Y$ axis represents the euclidean distance: each point $T\left(t_{1}, \ldots t_{n}\right)$ in the original $n \mathrm{D}$ space is associated in the dual space with a curve that represents the distance between $T$ and every point of $\Delta$.

In this dual space, the points $Q_{i}, i \in[1, n]$ are all represented by the same curve $L_{Q}^{0}$ since they are all equidistant from each point of $\Delta$ (Figure $5 \mathrm{~b}$ ). We consider that all the points $Q_{i}, i \in[1, n]$ are on the external border of the annulus, therefore $L_{Q}^{0}$ represents the external radius $\left(R_{e}\right)$ variation with respect to the center position. An annulus of width $\omega$ passing through the points $Q_{i}, i \in[1, n]$ is represented by a vertical segment of length $\omega$ having one of its endpoints on $L_{Q}^{0}$. The $X$ coordinate of the segment corresponds to the annulus center coordinate on $\Delta$.

The translation of $L_{Q}^{0}$ by $(0,-\omega)$ is denoted by $L_{Q}^{1}$ which represents the internal radius variation with respect to the center position.

In the dual space, an annulus $A$ of center $C\left(c^{\prime}, 0\right)$ with points $Q_{i}, i \in[1, n]$ on its external border corresponds to the vertical line segment $\left[L_{Q}^{0}\left(c^{\prime}\right), L_{Q}^{1}\left(c^{\prime}\right)\right]$ of length $\omega$.

a)

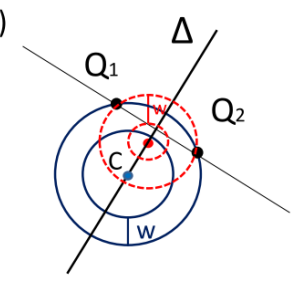

b)

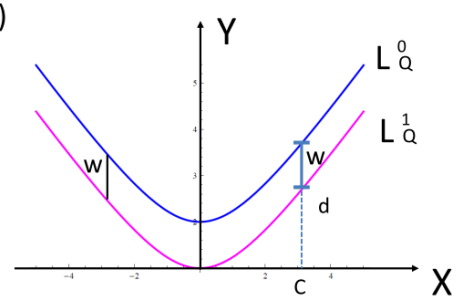

Fig. 5. a) The 2D annulus having $Q_{1}$ and $Q_{2}$ as border point has its centers on the bisector $\Delta$ of the line segment $Q_{1} Q_{2}$. b) All annuli for which $Q_{1}$ and $Q_{2}$ are on $B_{e}$ correspond to the set of all the vertical line segments of length $\omega$ having one of its endpoints on $L_{Q}^{0}$ and the other on $L_{Q}^{1}$.

For every point $T$ in the image, it is possible to see if it is inlier or outlier to an annulus of width $\omega$ centered on $C$ and having the $n$ points $Q_{i}$ on its external border by examining its associated curve $L_{T}$. The point $T$ is inlier if, in the dual space, $L_{T}$ intersects the vertical segment $\left[L_{Q}^{0}\left(c^{\prime}\right), L_{Q}^{1}\left(c^{\prime}\right)\right]$ with $c^{\prime}=\operatorname{dist}(C, B)$ since in this case it is between $L_{Q}^{0}\left(c^{\prime}\right)=R_{e}$ and $L_{Q}^{1}\left(c^{\prime}\right)=R_{i}$ (Figure 6). 


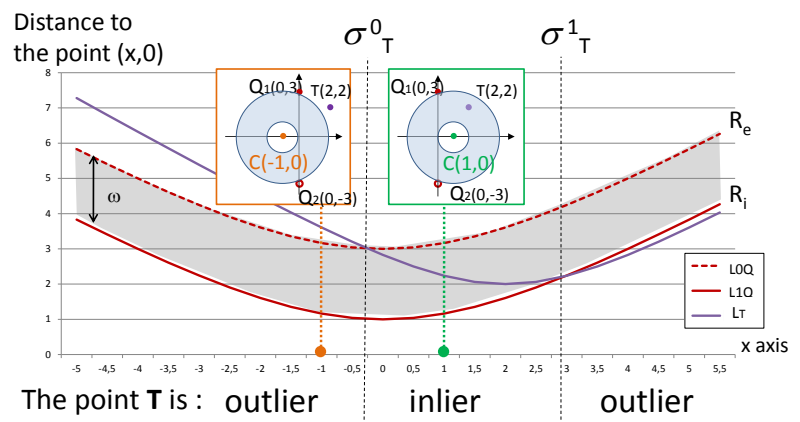

Fig. 6. 2 D example: the point $T$ represented in the dual space by the curve $L_{T}$ intersects the curve $L_{Q}^{0}$ at $\sigma_{T}^{0}$ and the curve $L_{Q}^{1}$ at $\sigma_{T}^{1}$. The point $T$ is thus inlier to the annulus having $Q_{1}$ and $Q_{2}$ on its external border between $\sigma_{T}^{0}$ and $\sigma_{T}^{1}$ and becomes outlier otherwise. The point $T$ is outlier to the annulus centered at $C(-1,0)$ and inlier to the one centered at $C(1,0)$.

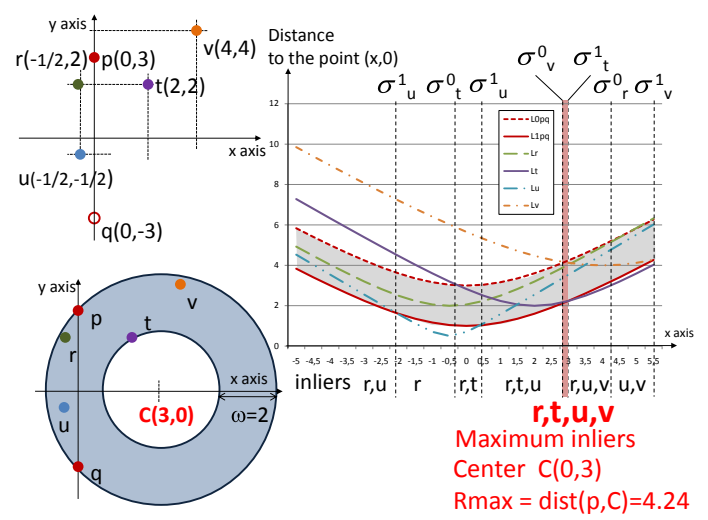

Fig. 7. $2 \mathrm{D}$ example: six points $p=Q_{1}, q=Q_{2}, r, u, v$, and $t$ in the primal space and their corresponding curves. The maximum number of inliers for an annulus having $p$ and $q$ on $B_{e}$ is reached when the center has an $x$-value around 3 .

\subsection{Finding the largest consensus set in a strip for a given $\left(Q_{1}, Q_{2}, \ldots, Q_{n}\right)$}

In order to know the number of inliers within any annulus defined by $n$ points $Q_{1} \ldots Q_{n}$, we check for every point $T$ in the image, the intersections $\sigma_{T}^{0}$ and $\sigma_{T}^{1}$ of $L_{T}$ with the strip boundaries $L_{Q}^{0}$ and $L_{Q}^{1}$. This check is important since any annulus corresponding to a vertical segment between the two intersections $\sigma_{T}^{0}$ and $\sigma_{T}^{1}$ in the strip always contains $T$ as an inlier; outside this interval, $T$ is always an outlier (Figure 6). 
When checking the intersections of every $L_{T}$ with $L_{Q}^{0}$ and $L_{Q}^{1}$, we use two values $f_{T}^{i}$ for $i=0,1$, which is set to 1 if $L_{T}$ enters the strip from $L_{Q}^{i}$, and -1 if $L_{T}$ leaves the strip from $L_{Q}^{i}$. Once the intersections $\sigma_{T}^{i}$, and the associated values $f_{T}^{i}$ for $i=0,1$ are calculated, we sort all the intersections in increasing order. As for determining the location(s) of the maximum number of inliers, a function $F(x)$ is used; initially we set $F(x)=n$ for every $x$, since we already know that $Q_{1}, \ldots Q_{n}$ are inliers. Then the value $f_{r}^{i}$ is added to $F(x)$ following the sorted order. By looking for the maximum value of $F(x)$, we obtain the center location(s) in the dual space corresponding to the maximum optimal consensus set(s). Figure 7 shows an example in $2 \mathrm{D}$ of this algorithm; the annulus in the primal space having $p=Q_{1}$ and $q=Q_{2}$ on its external border is optimal in terms of inliers at a center around 3 between $\sigma_{v}^{0}$ and $\sigma_{t}^{1}$ when all the dual curves are inside $L_{Q}^{0}$ and $L_{Q}^{1}$ (i.e. when all the points are inliers).

This procedure is repeated for all the combination of $n$ points in the image until finding the right center(s) of the annulus (annuli) having $n$ points on $B_{e}$ that maximizes the number of inliers. Since a sorting of complexity $O(N \log N)$ of the intersection is needed and since the algorithm is repeated for every couple of $n$ points, the final complexity is $O\left(N^{n+1} \log N\right)$.

\subsection{Algorithm}

Algorithm 1 gives an example of the $n \mathrm{D}$ annulus fitting algorithm. The inputs are a set $S$ of $n \mathrm{D}$ points and a width $\omega$. Output is a set $V$ of centers and radius of the best annuli.

\subsection{Degenerate cases}

There are degenerate cases that must be treated carefully when examining the number of inliers and outliers. They can be summarized as follows :

- Invalid radius : $L_{Q}^{1}$ represents the internal border of the annulus; when it is negative the annulus is not valid. An example of such invalid radius is seen in Figure 8a, in this figure $T$ becomes inlier at $\sigma_{T}^{1}$ and at $\sigma_{T}^{0}$, instead of $\sigma_{T}^{0}$ since at $\sigma_{T}^{0}$ the radius is negative.

- Intersection of $L_{T}$ with $L_{Q}^{0}$ and $L_{Q}^{1}$ : When both $L_{Q}^{0}$ and $L_{Q}^{1}$ are each intersected once we have the regular case explained in the Algorithm 1. The degenerate cases occur when one of the two curves is not intersected or when it is intersected twice. These cases can be explained as follows:

- $L_{T}$ and $L_{Q}^{0}$ have no common point: in this case the intersection of $L_{T}$ with $L_{Q}^{1}$ must be verified. If $L_{Q}^{1}$ is not intersected, we must check if $L_{T}$ is between the two curves, in this case $T$ is always inlier, otherwise $T$ is always outlier (Figure 8b). If $L_{Q}^{1}$ is intersected once, $L_{T}$ is inside the two curves and $T$ is always inlier (Figure 8c). If $L_{Q}^{1}$ is intersected twice, this means that $L_{T}$ is between the two curves and thus the point $T$ is inlier before the first intersection $\sigma_{T}^{1}$ of $L_{T}$ and after the second intersection $\sigma_{T}^{2}$ as seen in Figure 9a. 


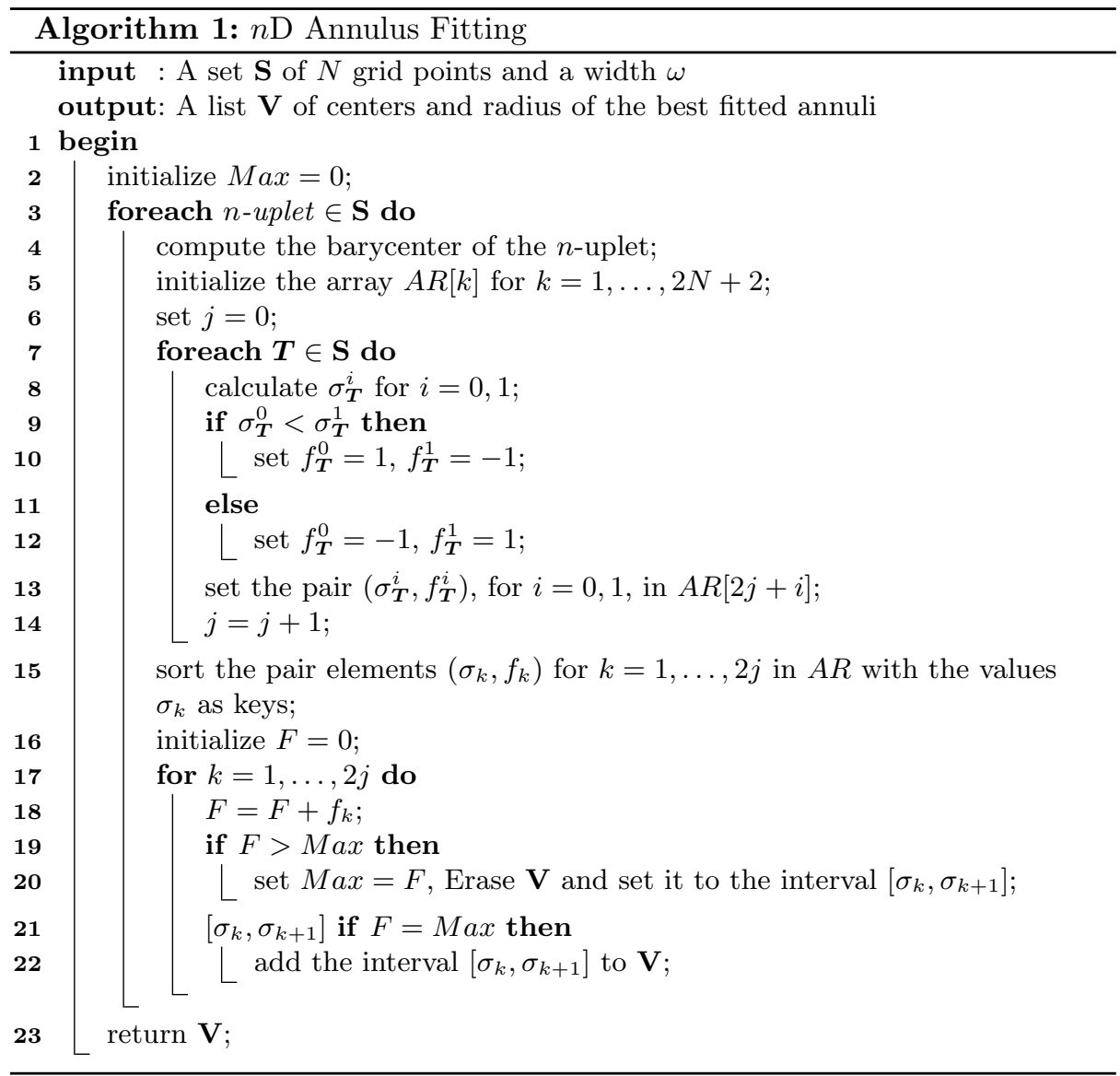

- $L_{T}$ has one intersection $\sigma_{T}^{0}$ with $L_{Q}^{0}$ : If $L_{T}$ has no intersection with $L_{Q}^{1}$, and $L_{T}$ is inside the two curves before the intersection $\sigma_{T}^{0}$, then $T$ is inlier before the intersection $\sigma_{T}^{0}$ and becomes outlier after this intersection (Figure $9 \mathrm{~b}$ ); otherwise $T$ is outlier before the intersection $\sigma_{T}^{0}$ and becomes inlier after this intersection.

If $L_{T}$ has two intersections with $L_{Q}^{1}$, then we have two cases: $T$ is inlier before the intersection $\sigma_{T}^{1}$ and between $\sigma_{T}^{2}$ and $\sigma_{T}^{0}$ and becomes outlier otherwise (Figure 9c) or $T$ is inlier between the two intersections $\sigma_{T}^{0}$ and $\sigma_{T}^{1}$ and after the intersection $\sigma_{T}^{2}$ and is outlier otherwise.

\section{Experiments}

This section presents the $2 \mathrm{D}$ and $3 \mathrm{D}$ experiments. 


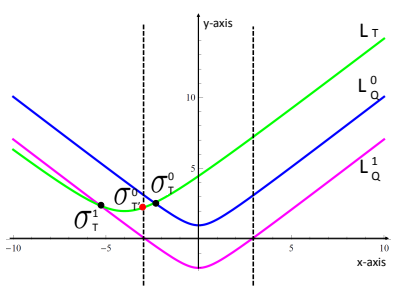

(a)

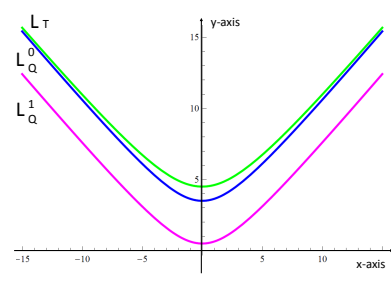

(b)

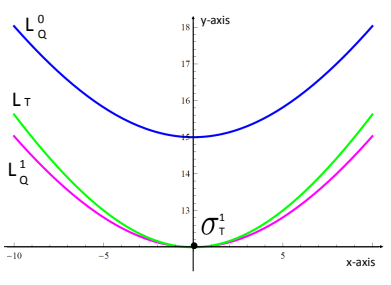

(c)

Fig. 8. Degenerate cases : a) The value of $L_{Q}^{1}$ is negative between the two vertical dashed lines; the point $T$ is inlier between $\sigma_{T}^{1}$ and $\sigma_{T /}^{0}$. b) $T$ is always outlier. c) $T$ is always inlier.

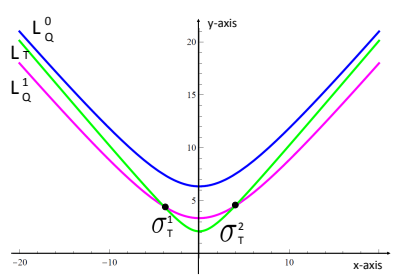

(a)

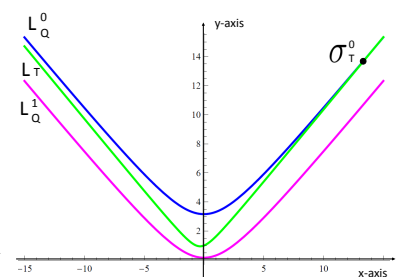

(b)

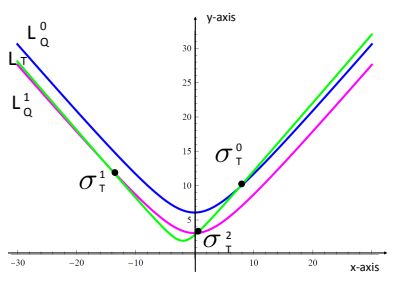

(c)

Fig. 9. Degenerate cases : a) $T$ is inlier before $\sigma_{T}^{1}$ and after $\sigma_{T}^{2}$. b) $T$ is inlier before $\sigma_{T}^{0}$. c) $T$ is inlier before $\sigma_{T}^{1}$ and between $\sigma_{T}^{2}$ and $\sigma_{T}^{0}$.

Table 1. The number of points and the optimal consensus set size.

\begin{tabular}{|c|l|l|c|c|l|}
\hline Figures & Number of points & Center & $\mathrm{R}$ & width & Opt. cons. set size \\
\hline Figure 10b & 646 & $(104.992,31.017)$ & 28.163 & 3 & 291 \\
Figure 11a & 225 & $(40.871,41)$ & 19.522 & 1 & 113 \\
Figure 11b & 65 & $(31.109,31.109)$ & 14.425 & 1 & 65 \\
Figure 12a & 1127 & $(51,51.008)$ & 26.992 & 6 & 1120 \\
Figure 12b & 56 & $(0.878,1.244,1.976)$ & 1.916 & 3 & 51 \\
Figure 13a & 109 & $(-4,-4,-4)$ & 2.742 & 1 & 90 \\
Figure 13b & 151 & $(0,0,0)$ & 6 & 3 & 116 \\
\hline
\end{tabular}

\subsection{Example for a 2D Real Image}

We tested our method on a real test image, as shown in Figure 10a, whose size is 140x69. Before applying our method, edge detection and mathematical 


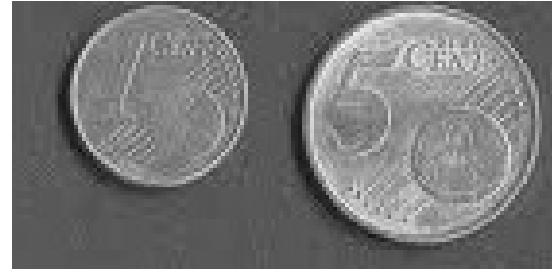

(a)

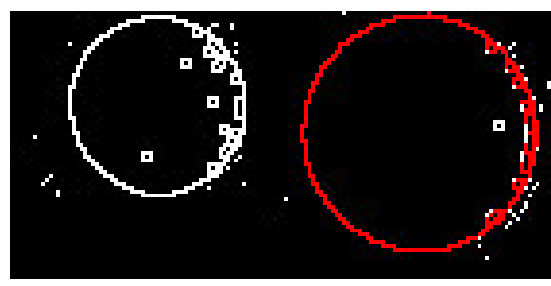

(b)

Fig. 10. An original image in a) and its optimal consensus set colored red in b).

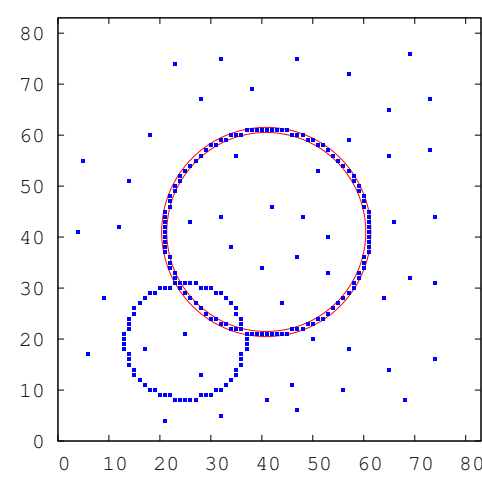

(a)

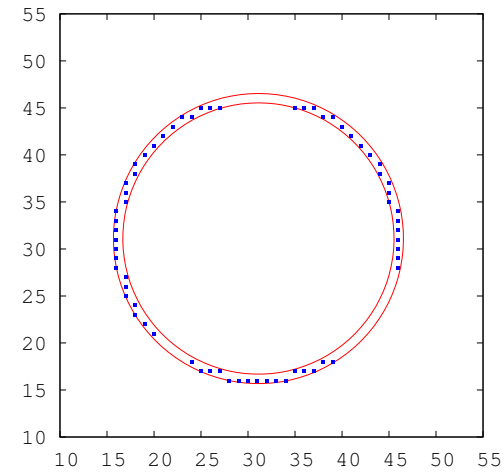

(b)

Fig. 11. a) Annulus fitting for a noisy digital Andres circle of width 1. b) Annulus fitting for a digital Andres circle of width 1.

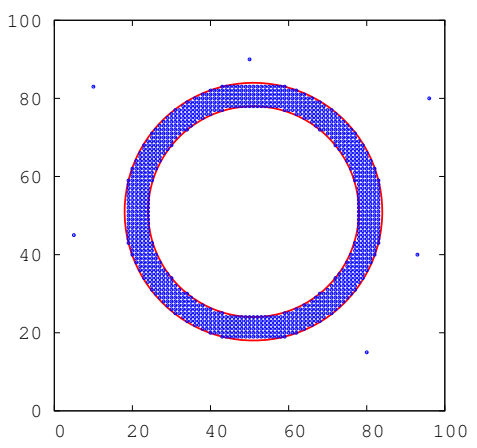

(a)

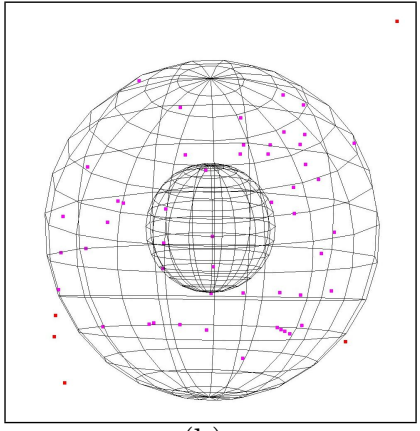

(b)

Fig. 12. a) Annulus fitting for a noisy digital Andres circle of width 6. b) Annulus fitting for noisy 3D data; a width $w=3$ is used. 


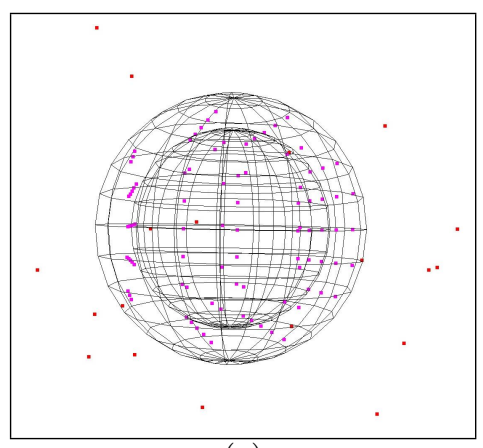

(a)

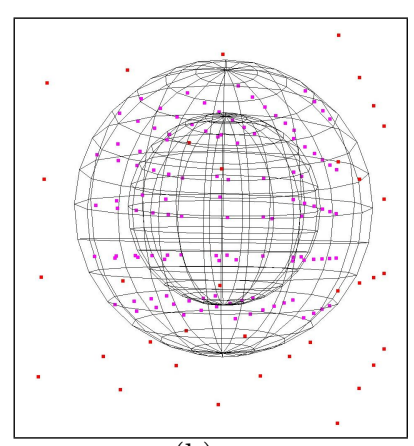

(b)

Fig. 13. a) Annulus fitting for noisy $3 \mathrm{D}$ data; a width $w=1$ is used. b) Annulus fitting for noisy $3 \mathrm{D}$ data; a width $w=3$ is used.

morphological filtering have been performed on the image; the number of points in the image after this preprocessing is 646 points. Our method is then applied to fit an annulus to the set of points. Figure $10 \mathrm{~b}$ shows the optimal consensus set, which includes 291 inliers. The width of the annulus is fixed to 3 .

\subsection{Example on 2D noisy images}

We then applied our method for 2D noisy digital Andres circles (points for Andres circles and noise are generated randomly for $2 \mathrm{D}$ as well as for $3 \mathrm{D}$ experiments) as shown in Figure 11a, 11b, 12a. For each of these set of points, an annulus of width $\omega=1, \omega=1$ and $\omega=6$ is used respectively. Table 1 show the number of points, the optimal consensus set size as well as the center position and the radius $R$ of the inner circle obtained after the fitting.

\subsection{D noisy images}

We applied our method for 3D noisy digital Andres spheres as shown in Figure 12a, 13a, 13b. For each of these set of points, an annulus of width $\omega=3$, $\omega=1$ and $\omega=3$ is used respectively. The last three lines of Table 1 shows the number of points, the optimal consensus set size as well as the center position and the radius $R$ of the inner sphere obtained after the fitting.

\section{Conclusion and Perspectives}

In this paper we have proposed a new approach for fitting $n \mathrm{D}$ annulus to a set of points while fixing the width of the annulus. The main advantage of our approach is that it guarantees optimal and exhaustive results from the point of view of the optimal (maximal) consensus set: we are guaranteed to fit an annulus with the least amount of outliers. We are also guaranteed to find all the optimal 
consensus sets. One of the future works concerns conic fitting such as ellipse, parabola and hyperbola. We also plan to implement a fast $2 \mathrm{D} / 3 \mathrm{D}$ algorithm for fitting annulus; such algorithm does not guarantee optimality but guarantees local maximality of inliers in the sense of the set inclusion and thus has a lower time complexity. We also plan to adapt the $n \mathrm{D}$ characterization of annulus for $k-$ Flake digital hyperspheres [18] as an extension of $[4,11]$.

\section{Acknowledgments}

The authors express their thanks to Mr. Pierre Boulenguez, who contributed in the implementation of a part of the 3D Fitting. The work for this paper was partly financed by Egide, franco-Japanese PHC Sakura project $n^{\circ} 27608 \mathrm{XJ}$ and by the Poitou Charentes region project $n^{o}$ 11/RPC-R-051.

\section{References}

1. P.K. Agarwal, S. Har-Peled, and K.R. Varadarajan. Approximating extent measures of points. Journal of the ACM, 51(4):606-635, 2004.

2. R. Agarwal, J. Gehrke, D. Gunopulos, and P. Raghavan. Automatic subspace clustering of high dimensional data for data mining applications. In Proceeding SIGMOD '98 international conference on Management of data, pages 94-105, 1998.

3. E. Andres and M.-A Jacob. The discrete analytical hyperspheres. IEEE Transactions on Visualization and Computer Graphics, 3:75-86, 1997.

4. E. Andres, G. Largeteau-Skapin, R. Zrour, A. Sugimoto, and Y. Kenmochi. Optimal consensus set and preimage of 4-connected circles in a noisy environment. In 21st International Conference on Pattern Recognition (ICPR 2012), pages 37743777. IEEE Xplore, 2012.

5. M. De Berg, P. Bose, D. Bremner, S. Ramaswami, G. Ramaswami, and G. Wilfong. Computing constrained minimum-width annuli of point sets. Algorithms and Data Structures, Lecture Notes in Computer Science, 1272:392-401, 1997.

6. J.M. Diaz-Banez, F. Hurtado, H. Meijer, D. Rappaport, and T. Sellares. The largest empty annulus problem. In Proceedings of the International Conference on Computational Science-Part III, volume 2331, 3, pages 46-54, Amsterdam, Holland, 2002. LNCS, Springer Verlag.

7. J. Dunagan and S. Vempala. Optimal outlier removal in high-dimensional spaces. Journal of Computer and System Sciences, 68(2):335-373, 2004.

8. M.A. Fischler and R.C. Bolles. Random sample consensus: A paradigm for model fitting with applications to image analysis and automated cartography. Communications of the ACM, 24:381-395, 1981.

9. S. Har-Peled and Y. Wang. Shape fitting with outliers. SIAM Journal on Computing, 33(2):269-285, 2004.

10. C. Kimme, D. Ballard, and J. Sklansky. Finding circles by an array of accumulators. Communication of the ACM, 18(2), 1975.

11. G. Largeteau-Skapin, R. Zrour, and E. Andres. $\mathrm{O}\left(n^{3} \log n\right)$ time complexity for the optimal consensus set computation for 4-connected digital circles. In 17-th International Conference on Discrete Geometry for Computer Imagery (DGCI13), volume 7749, pages 241-252, Sevilla, Spain, 2013. LNCS, Springer Verlag. 
12. J. Matousek. On enclosing $\mathrm{k}$ points by a circle. Information Processing Letters, 53(4):217-221, 1995.

13. J. O'Rourke, S.R. Kosaraju, and N. Megiddo. Computing circular separability. Discrete and Computational Geometry, 1:105-113, 1986.

14. M.S. Phan, Y. Kenmochi, A. Sugimoto, H. Talbot, E. Andres, and R. Zrour. Efficient robust digital annulus fitting with bounded error. In 17-th International Conference on Discrete Geometry for Computer Imagery (DGCI13), volume 7749, pages 253-264, Sevilla, Spain, 2013. LNCS, Springer Verlag.

15. Stephen M. Robinson. Fitting spheres by the method of least squares. Commun. ACM, 4(11):491-, November 1961.

16. T. Roussillon, L. Tougne, and S. Sivignon. On three constrained versions of the digital circular arc recognition problem. In 15-th International Conference on Discrete Geometry for Computer Imagery (DGCI09), pages 34-45. LNCS, Springer Verlag, 2009.

17. M. Smid and R. Janardan. On the width and roundness of a set of points in the plane. International Journal of Computational Geometry, 9(1):97-108, 1999.

18. J.L. Toutant, E. Andres, and Tristan Roussillon. Digital circles, spheres and hyperspheres: From morphological models to analytical characterizations and topological properties. Discrete Applied Mathematics, 161(16-17):2662-2677, 2013.

19. R. Zrour, Y. Kenmochi, H. Talbot, L. Buzer, Y. Hamam, I. Shimizu, and A. Sugimoto. Optimal consensus set for digital line and plane fitting. International Journal of Imaging Systems and Technology, 21(1):45-57, 2011.

20. R. Zrour, G. Largeteau-Skapin, and E. Andres. Optimal consensus set for annulus fitting. In 16th International Conference on Discrete Geometry for Computer Imagery (DGCI11), volume 6607, pages 358-368, Nancy, France, 2011. LNCS, Springer Verlag. 